\title{
Metabolic response of Escherichia coli to subinhibitory concentration of ofloxacin
}

\author{
Engin KOÇAK ${ }^{1 *}$ (D), Ceren ÖZKUL ${ }^{2}$ (D) \\ 1 Department of Analytical Chemistry, Faculty of Pharmacy, Hacettepe University, Ankara, Turkey. \\ 2 Department of Pharmaceutical Microbiology, Faculty of Pharmacy, Hacettepe University, Ankara, Turkey. \\ * Corresponding Author. E-mail: kengin@hacettepe.edu.tr (E.K.); Tel. +90-312-305-1499
}

Received: 15 November 2019 / Revised: 29 April 2020 / Accepted: 05 May 2020

ABSTRACT: Antibiotic resistance is one of the most important problems worldwide. In order to overcome antibiotic resistance, pathogen-antibiotic interactions should be investigated at molecular level. In our present study, we focused on metabolic differences in Escherichia coli (E. coli) under ofloxacin stress to understand adaptation and resistance mechanisms. We used GC/MS based metabolomics approach for metabolite identification and quantification. Experimental results showed that 17 metabolites were altered under subinhibitory concentration of ofloxacin. Pathway analysis indicated that TCA cycle, glyoxylate and dicarboxylate metabolism, biosynthesis of fatty acids, glutathione metabolism were induced by ofloxacin. Moreover, we evaluated metabolites individually and we found that spermine and L-ascorbic acid increased in ofloxacin treated groups. These metabolites are important in resistance and detoxifying processes in cells. In conclusion, altered metabolome profile gave information on adaptation and resistance processes in $E$. coli against ofloxacin. These metabolic changes will help to enlighten resistance mechanisms and discover novel treatment strategies against the pathogens.

KEYWORDS: Metabolomics; antibiotic resistance; GC/MS; MS-DIAL; TCA cycle.

\section{INTRODUCTION}

Antibiotic resistance is one of the most important health issues around the world. There is growing effort worldwide to discover new antibiotics and overcome evolving antimicrobial resistance. Synthetic or natural molecules have been investigated for their inhibitory effects on microbial metabolism. However, classical antibiotic discovery approaches are not sufficient to respond increasing resistance problem [1,2].

Developing analytical techniques and bioinformatics offer great opportunity to analyze cellular processes at molecular levels. Genomics, transcriptomics, proteomics and metabolomics have been used to define disease pathogenesis, find diagnostic and prognostic biomarkers, and understand mode of action of drug molecules [3-5]. Omics technologies have been drawn attention in microbiology to analyze genomic and post genomic structure of bacterial species. Moreover, these techniques have been used to understand relationship between pathogens and antimicrobial agents at molecular level. The results have showed essential information about changing of genotype and phenotype of pathogens under antibiotic stress conditions [6,7]. In addition, antibiotic resistant and sensitive samples have been investigated via omics technologies to find molecular and genetic differences [8,9]. This systematic approach could be a key tool to discover new antibiotics. Especially understanding of antimicrobial resistance mechanisms offers new routes to overcome antibiotic resistance problem. However, in recent years researches focused on mostly genetic and transcriptional regulatory network regarding antibiotic resistance, metabolic perturbation after treatment was missing part to understand mode of action of antibiotic and resistance machinery. Metabolites in organisms are side or end products of biological processes and their amounts altered with genetic and physiological events [10]. Metabolome structure in cells is very dynamic based on changes in the transcriptome and proteome. Metabolite profiling gives comprehensive information about the organism under different conditions [11]. In microbiological settings, metabolomics techniques are increasing popularity to observe metabolic changes induced by antibiotics and resistant mechanism of pathogens against antibiotics [12,13]. 
Currently, there is no definitive or standardized operating procedure for metabolomics. Various analytical techniques have been developed for extraction and detection of metabolites. Among these techniques, mass spectroscopy is well-established analytical system for metabolomics. It offers high sensitivity and resolution. Moreover, mass spectroscopy could be combined with separation systems (gas and liquid chromatography). Gas chromatography-mass spectroscopy (GC/MS) is the standardized method for primary metabolite analysis. GC/MS system allows for the identification and quantification of volatile and thermally stable compounds. Due to the high reproducibility, resolution and the existence of libraries of mass spectra, it is called gold standard for metabolomics research [14].

Ofloxacin is a member of the fluoroquinolone group and using in veterinary and human medicine. Ofloxacin has a broad-spectrum antibacterial effect against Gram negative, Gram positive and anaerobic bacteria [15]. It inhibits DNA gyrase and topoisomerase IV, which are involved in DNA replication. The typical resistance mechanism against ofloxacin is alterations of the target enzymes (DNA gyrase and topoisomerase IV) by chromosomal mutations [16]. However, metabolic perturbations under ofloxacin stress needs to be explained in order to understand the adaptation and resistance processes against the ofloxacin and floroquinolone antibiotics.

In our present study, we used GC/MS based metabolomics approach to observe metabolic changes in E. coli under ofloxacin stress. Our aim was to understand adaptation and resistance mechanism against ofloxacin at metabolite level. We found statistically altered metabolites between control and ofloxacin treated groups. We evaluated metabolites via pathway analysis and also individually. Our results showed that various pathways were deregulated with sublethal doses of ofloxacin. We believe that our results will contribute to develop new treatment method against the E.coli.

\section{RESULTS}

The MIC value of ofloxacin against E. coli ATCC 25922 was $0.02 \mu \mathrm{g} / \mathrm{mL}$, which was within the expected susceptibility range. Thus the half of the MIC value $(0.01 \mu \mathrm{g} / \mathrm{mL})$ was used for further metabolomics studies.

In metabolomics analysis, metabolites were extracted in methanol/water mixture and analyzed in GC/MS system. Metabolites were separated in GC system and analyzed in MS part. Base peak chromatograms (BPC) were given in Figure 1 for control and ofloxacin treated groups.

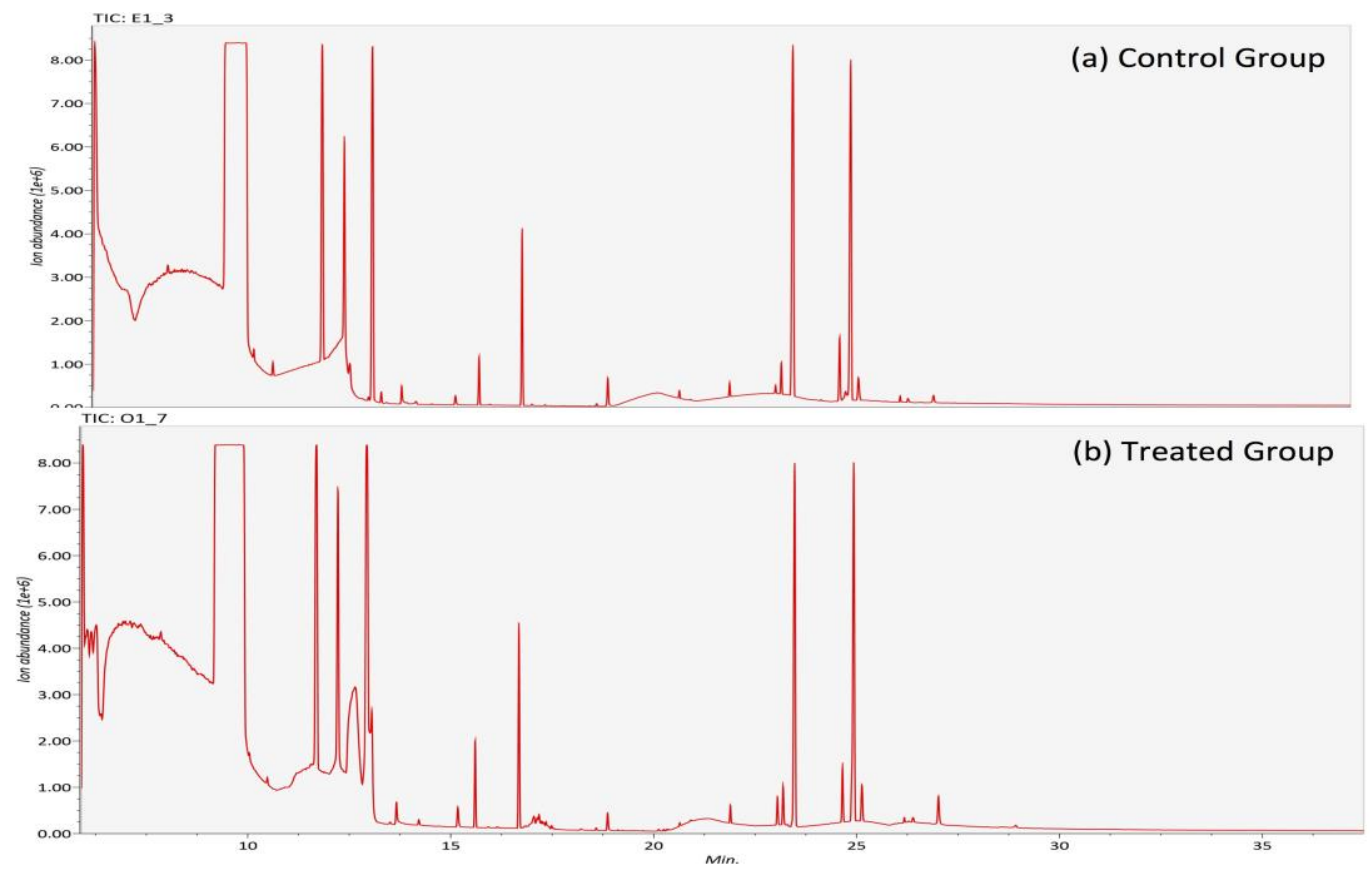

Figure 1. Base peak chromatograms of control and ofloxacin treated groups.

Raw GC/MS data were processed in MS-DIAL software. We detected 593 peaks and identified 131 metabolites. Peak intensities were calculated and normalized according to total ion chromatogram (TIC). 
Principal component analysis (PCA) was used to observe effect of ofloxacin over global metabolome structure of E. coli (Figure 2).

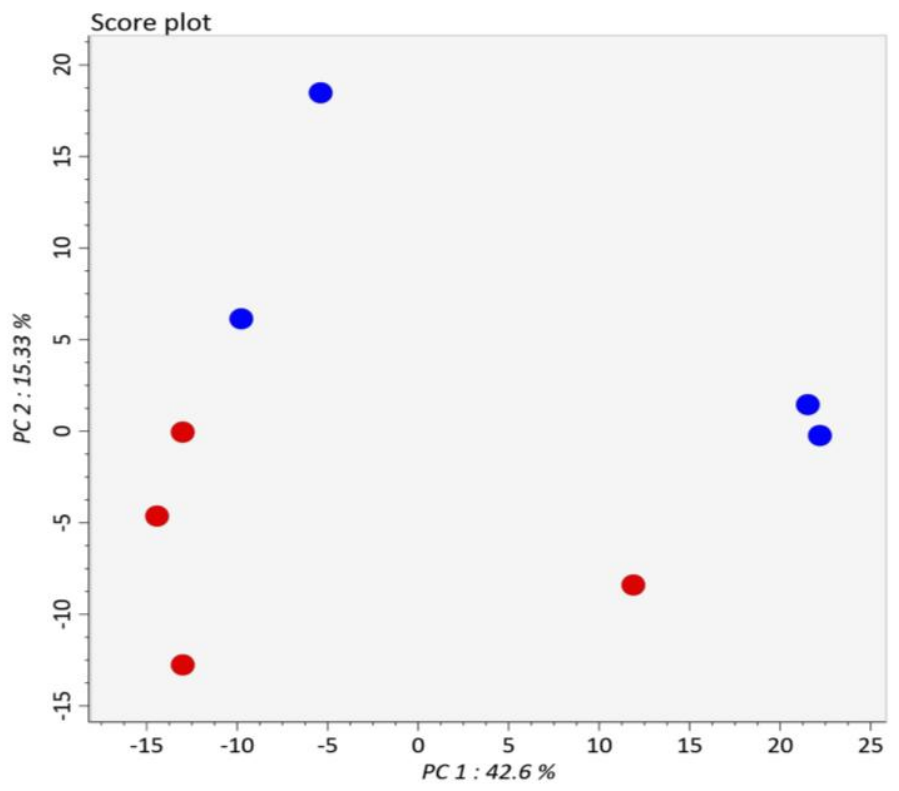

Figure 2. Principal component analysis of control (blue) and ofloxacin treated (red) groups to observe general effect of ofloxacin over metabolome structure.

Identified metabolites were evaluated statistically to find altered metabolites under ofloxacin stress. In statistical analysis, we used two-sample t-test $(\mathrm{P}$ values $<0.05$ ). We focused on significant metabolites (fold change $>1.5$ ) for reliable analysis. We found that 17 metabolites were altered under ofloxacin stress. 10 metabolites increased and 7 metabolites decreased. Altered metabolites were listed in Table 1.

Table 1. Altered metabolites under ofloxacin stress

\begin{tabular}{lccc}
\hline Upregulated Metabolites & $\begin{array}{c}\text { Fold-Change } \\
\text { (Control/Treated) }\end{array}$ & $\begin{array}{c}\text { Downregulated } \\
\text { Metabolites }\end{array}$ & $\begin{array}{c}\text { Fold-Change } \\
\text { (Control/Treated) }\end{array}$ \\
\hline Glycine & 0.046 & Beta-Alanine & 1.895 \\
Aconitic acid & 0.061 & Tyramine & 1.994 \\
L-Ascorbic acid & 0.070 & Isocitric acid & 2.25 \\
Palmitic acid & 0.070 & Stearic acid & 2.535 \\
Cellobiose & 0.126 & Maleic acid & 4.201 \\
Niacinamide & 0.336 & Oxamic acid & 7.564 \\
Spermine & 0.337 & Malate & 13.665 \\
Methionine sulfoxide & 0.376 & & \\
Succinic acid & 0.462 & & \\
N-acetyl-5- & 0.642 & & \\
hydroxytryptamine & & & \\
\hline
\end{tabular}

Altered metabolites were analyzed in metaboanalyst platform to find systematic effect of ofloxacin over cellular pathways (Figure 3). We evaluated pathways for their statistical significance and pathway impact. Tricarboxylic acid cycle (TCA cycle), beta alanine metabolism, glyoxylate and dicarboxylate metabolism, and propanoate metabolism was affected by ofloxacin. Moreover, we used KEGG pathway database and found that altered metabolites were also involved in different pathways such as Glutathione metabolism, Nicotinate and nicotinamide metabolism, Tyrosine metabolism (Table 2). In addition, it was observed that ofloxacin induced expression level of various fatty acid synthesis, which are palmitic and stearic acids. 


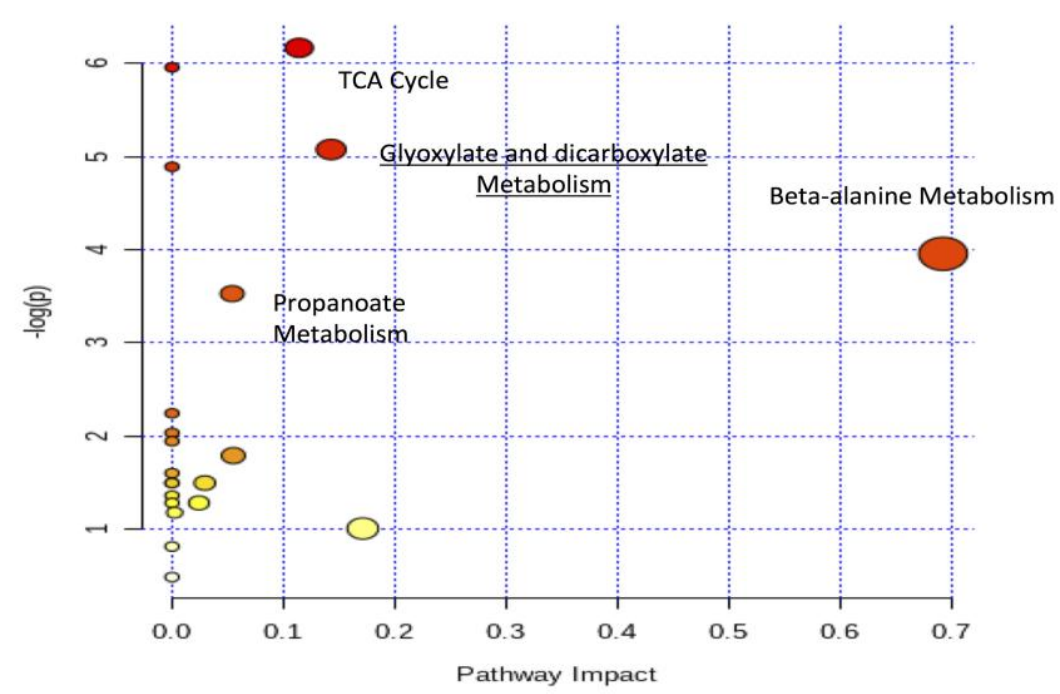

Figure 3. Pathway analysis of differentially expressed metabolites in metaboanalyst platform.

Table 2. Ofloxacin induced pathways in E. coli (KEGG database)

\begin{tabular}{ll}
\hline No & Metabolic pathway (numbers of metabolites in pathway) \\
\hline $\mathbf{1}$ & eco01100 Metabolic pathways - Escherichia coli K-12 MG1655 (13) \\
$\mathbf{2}$ & eco01110 Biosynthesis of secondary metabolites - Escherichia coli K-12 MG1655 (8) \\
$\mathbf{3}$ & eco01120 Microbial metabolism in diverse environments - Escherichia coli K-12 \\
& MG1655 (7) \\
$\mathbf{4}$ & eco01130 Biosynthesis of antibiotics - Escherichia coli K-12 MG1655 (5) \\
$\mathbf{5}$ & eco00630 Glyoxylate and dicarboxylate metabolism - Escherichia coli K-12 MG1655 (5) \\
$\mathbf{6}$ & eco01200 Carbon metabolism - Escherichia coli K-12 MG1655 (4) \\
$\mathbf{7}$ & eco00020 Citrate cycle (TCA cycle) - Escherichia coli K-12 MG1655 (4) \\
$\mathbf{8}$ & eco00760 Nicotinate and nicotinamide metabolism - Escherichia coli K-12 MG1655 (3) \\
$\mathbf{9}$ & eco00350 Tyrosine metabolism - Escherichia coli K-12 MG1655 (3) \\
$\mathbf{1 0}$ & eco00480 Glutathione metabolism - Escherichia coli K-12 MG1655 (3) \\
$\mathbf{1 1}$ & eco00680 Methane metabolism - Escherichia coli K-12 MG1655 (3) \\
\hline
\end{tabular}

\section{DISCUSSION}

In the present work, we focused on metabolic perturbations in E. coli under ofloxacin stress. GC/MS based metabolomics approach was used to analyze metabolic differences. We identified total of 131 metabolites, calculated peak areas and compared two experimental groups (control and ofloxacin treated groups). PCA analysis showed that metabolome structures of control and ofloxacin treated groups was quite different (Figure 2). This result indicated that ofloxacin-induced stress affects overall metabolome structure. Altered metabolites were found with statistical analysis (t-test) and 17 metabolites were differentially expressed ( $\mathrm{p}<0.05$ and fold change $>1.5$ ). While 10 metabolites increased, 7 metabolites decreased with the effect of ofloxacin. Altered metabolites were evaluated in Metaboanalyst and KEGG pathway database to observed systematic changes in E. coli.

\subsection{Ofloxacin causes deregulation in energy metabolism}

In present work, It was observed the dsyregulation in TCA cycle, which is the main step in energy production and biosynthetic precursor synthesis. In recent studies, it was showed that deregulation in TCA cycle could be activator of resistance and adaptation processes against antibiotics. Rosato et. al. [19] showed that TCA cycle produces more reactive oxygen species under antibiotic stress and this may be SOS signal for detoxification and repair. In accordance with this study, we observed deregulation in TCA cycle under ofloxacin induced stress. Aconitic acid, succinic acid (succinate), isocitric acid (isocitrate) and malate were 
altered with the effect of ofloxacin (Table 3). Aconitic acid and succinic acid increased 16.66 and 2.17 fold. Also Malate and isocitrate decreased 13.66 and 2.25 fold. The deregulation in TCA cycle may be evaluated as the initial mechanism for resistance in E. coli.

Table 3. Altered metabolites in TCA cycle.

\begin{tabular}{lcccc}
\hline Metabolites & $\begin{array}{c}\text { Normalized Peak } \\
\text { intensity control } \\
\text { group }\end{array}$ & $\begin{array}{c}\text { Normalized } \\
\text { peak intensity } \\
\text { ofloxacin } \\
\text { treated group }\end{array}$ & $\begin{array}{c}\text { Control/ } \\
\text { Treated group }\end{array}$ & P value \\
\hline $\begin{array}{l}\text { Aconitic acid } \\
\text { (Aconitate) }\end{array}$ & 0.00023 & 0.0038 & 0.06 & $7.25 \times 10^{-5}$ \\
\hline $\begin{array}{l}\text { Succinic acid } \\
\text { (Succinate) }\end{array}$ & $7.41 \times 10^{-5}$ & 0.00016 & $\begin{array}{c}0.46 \\
\text { (upregulated) }\end{array}$ & 0.0329 \\
\hline $\begin{array}{l}\text { Isocitric acid } \\
\text { (Isocitrate) }\end{array}$ & $1.86 \times 10^{-5}$ & $8.25 \times 10^{-6}$ & $\begin{array}{c}2.25 \\
\text { (Downregulated) }\end{array}$ & 0.0473 \\
\hline Malate & $9.65 \times 10^{-5}$ & $7.0 \times 10^{-6}$ & $\begin{array}{c}13.66 \\
\text { (Downregulated) }\end{array}$ & $1.77 \times 10^{-5}$ \\
\hline
\end{tabular}

Glyoxylate cycle is a variation of TCA cycle in energy metabolism. The glyoxylate cycle has a role on the conversion of acetyl-CoA to succinate for the synthesis of carbohydrates. Various studies showed that glyoxylate cycle was upregulated under oxidative stress and had an important role in pathogenesis [20,21]. In general, bactericidal antibiotics lead increased oxidative stress [22]. Nadakumar et. al. showed that glyoxylate cycle is upregulated in Mycobacterium tuberculosis under various antibiotics [23]. Ahn et. al. investigated glyoxylate shunt via transcriptomics under oxidative stress conditions. They observed that glyoxylate cycle had a crucial role on tolerating oxidative stress and cell survival. However, the mechanism is still not clear [24]. In our present study, we found that most of the metabolites (Glycine, succinic acid, aconitic acid) in Glyoxylate and dicarboxylate metabolism increased under ofloxacin stress. This result showed that ofloxacin might induce oxidative stress in E. coli and Glyoxylate and dicarboxylate metabolism upregulated to protect cells against oxidative stress with unknown mechanisms.

In addition, we found that some biological processes like pyruvate and propanoate metabolism in energy production was also affected by ofloxacin. In further experiments, we will focus on energy metabolism under antibiotic stress conditions with targeted metabolomics.

\subsection{Glutathione metabolism was upregulated in ofloxacin treated group}

Due to the production of high levels of reactive oxygen species (ROS), which may be induced by bactericidal antibiotics, the cellular antioxidant mechanism plays critical role in antibiotic resistance. Glutathione is the key modulator in defense system against ROS species and very important for cell growth and survival. In previous studies, it was showed that glutathione plays important role in antibiotic resistance process $[25,26]$. In our present work, we found that three metabolites, which are involved in glutathione metabolism, were increased in ofloxacin treated groups. Glycine is the essential material for biosynthesis of glutathione. According to our present findings, glycine was increased up to 21.74 times in ofloxacin treated groups. Moreover, other metabolites, L-ascorbic acid and spermine, which are involved in glutathione metabolism significantly increased (Table 4). L-ascorbic acid has also antioxidant properties and has a crucial role in cellular system.

Spermine, which is in glutathione metabolism, is a member of polyamines, which are polycationic molecules, produced through complex pathways. Polyamines involve in a wide range of biological processes. In recent studies, the interactions between polyamines and porins were defined. Porin proteins are main regulators for overall permeability of the outer membrane. Lyer et.al showed that four polyamines (spermine, spermidine, cadaverine and putrescine) were very effective on the activity of E. coli porins OmpC and $\mathrm{OmpF}$ [27]. These polyamines suppress channel opening and promote the inactive state. Saraty et. al. also evaluated the effects of polyamines on porins. They found that polyamines decreased membrane permeability of the outer membrane, which leads to decreased intake of fluoroquinolones in Mycobacteria [28]. Increased levels of spermine in our study may lead to membrane based-resistance against ofloxacin. 
Table 4. Altered metabolites in glutathione metabolism.

\begin{tabular}{lcccc}
\hline Metabolites & $\begin{array}{c}\text { Normalized peak } \\
\text { intensity Control } \\
\text { Group }\end{array}$ & $\begin{array}{c}\text { Normalized } \\
\text { peak intensity } \\
\text { ofloxacin } \\
\text { treated group }\end{array}$ & $\begin{array}{c}\text { Control / } \\
\text { Treated group }\end{array}$ & P value \\
\hline Glycine & $6.78 \times 10^{-6}$ & 0.00014 & $\begin{array}{c}0.046 \\
\text { (Upregulated) }\end{array}$ & 0.028 \\
\hline Ascorbic acid & $6.05 \times 10^{-6}$ & $8.68 \times 10^{-5}$ & $\begin{array}{c}0.070 \\
\text { (Upregulated) }\end{array}$ & $4.6 \times 10^{-6}$ \\
\hline Spermine & $4.18 \times 10^{-5}$ & 0.000124 & $\begin{array}{c}0.337 \\
\text { (Upregulated) }\end{array}$ & 0.040 \\
\hline
\end{tabular}

\subsection{Fatty acid synthesis was affected by ofloxacin}

In present work, it was observed that biosynthesis of unsaturated fatty acid in E. coli was induced by ofloxacin. We found that decreased levels of stearic acid and increased levels of palmitic acid. It has been well known that lipid composition is very important parameter in antibiotic resistance. Fatty acids are major components of phospholipids and membrane structure, which are essential for cell metabolism. In recent studies, it was showed that fatty acids could be a potential target for antimicrobial agents [29,30]. Furthermore, Dunnict et. al showed that fatty acid composition was different in gram negative antibiotic resistant strains [31]. Kulukina et. al showed that fatty acid and lipid composition involved in nonspecific antibiotic resistance [32]. Li et. al. analyzed metabolome structure of E. coli under ciprofloxacin stress. They found that ciprofloxacin increased the fatty acid composition and decreased stearic acid levels in cells [6]. Changes in fatty acid composition could be important in adaptation process against stress condition induced by ofloxacin.

\subsection{Nicotine and nicotinamide metabolism}

Nicotinate and nicotinamide metabolisms were also induced by ofloxacin. Niacinamide (Nicotinamide) is the amide derivative of nicotinic acid and essential for organisms as the precursors for generation of coenzymes, NAD+ and NADP+, which utilizes the redox reactions and carry electrons from one reaction to another. They therefore exist in oxidized $(\mathrm{NAD}(\mathrm{P})+)$ and reduced $(\mathrm{NAD}(\mathrm{P}) \mathrm{H})$ forms. These coenzymes are crucial for many metabolic pathways including glycolysis, TCA cycle, pentose phosphate cycle, fatty acid biosynthesis and metabolism pathways and many others. In the literature, there is no report on the significant role of nicotinamide in antibiotic resistance. In further studies, we will focus on this pathway and its interaction with other pathways.

\section{CONCLUSION}

In our present study, we focused on metabolic perturbation in E. coli under ofloxacin stress. The metabolite profiling represents the downstream effect of an organism's genome and its interaction with the environment. This is especially important to understand resistance and adaptation of pathogens under stress conditions. We found changes in various metabolic pathways including TCA cycle, Glyoxylate and dicarboxylate metabolism, and fatty acid biosynthesis and glutathione metabolism. These metabolic pathways are very important in antibiotic resistance and adaptation processes and related ofloxacin induced toxicity. In further experiments we will focus on these pathways to use ofloxacin more efficiently. We believe that these results will contribute to literature in order to enlight development of antibiotic resistance in $E$. coli.

\section{MATERIALS AND METHODS}

\subsection{Bacterial culture}

E. coli ATCC 25922 was subcultured on Tryptic Soy Agar. In order to confirm the minimum inhibitory concentration (MIC) value of ofloxacin against E. coli, standard broth microdilution was performed according to the method reported by the Clinical Laboratory Standards Institute (CLSI) [17]. The MIC value of ofloxacin was determined and compared with the latest breakpoint tables of EUCAST. For proteomics 
experiments, subinhibitory concentration (half of the MIC) ofloxacin was used. E. coli ATCC 25922 was cultured on Mueller Hinton Broth (MHB) and incubated under $37^{\circ} \mathrm{C}$ until the log phase was achieved. The bacterial suspension was prepared using MHB that contained the MIC/2 value of ofloxacin to obtain a bacterial concentration of $5 \times 10^{5} \mathrm{cfu} / \mathrm{mL}$ bacteria. The same concentration of bacterial culture under same conditions was also prepared without adding any antibiotic. Flasks were incubated at $37^{\circ} \mathrm{C}$ for $20 \mathrm{~h}$. Experiments were performed with four replicates.

\subsection{Extraction of metabolites}

After centrifugation the cell pellets were washed by phosphate buffer saline (PBS) twice. The cells were enzymatically disrupted by using lysis buffer. In metabolites extraction, we used methanol (Sigma): water co solvent system (9:1 v/v). $850 \mu \mathrm{L}$ extraction solvent added to $150 \mu \mathrm{L}$ sample and mixed in a vortex for $1 \mathrm{~min}$. Proteins were participated with the effect of methanol. Samples were centrifuged at $15000 \mathrm{~g}$ for 10 minutes. Supernatant were taken and carefully evaporated under vacuum centrifuge. The derivatization process was carried out in two steps. In first step, $20 \mu \mathrm{L}$ methoxyamine solution in pyridine $(20 \mathrm{mg} / \mathrm{mL})$ (Sigma) was added to the dried extract and incubated at $70{ }^{\circ} \mathrm{C}$ for $90 \mathrm{~min}$. In second step, $100 \mu \mathrm{L}$ of MSTFA (sigma) was added and incubated at $37^{\circ} \mathrm{C}$ for 30 minutes. After derivatization, samples were transferred to auto sampler vials for GC/MS analysis.

\subsection{Chromatographic and mass spectrometry conditions}

Gas chromatography-mass spectrometry (GC-MS)-based metabolomics analysis was performed as described previously [18]. Metabolites were analyzed using GC-MS (Shimadzu GCMS-QP2010 Ultra) with a DB-5MS stationary phase column $(30 \mathrm{~m}+10 \mathrm{~m}$ DuraGuard $\times 0.25 \mathrm{~mm}$ i.d. and $0.25-\mu \mathrm{m}$ film thickness). Helium was used as the carrier gas at $1 \mathrm{~mL} / \mathrm{min}$ flow rate. Samples were injected in non-split mode. Injection volume was adjusted at $2 \mu \mathrm{L}$. The injector temperature was held at $250{ }^{\circ} \mathrm{C}$. In gradient system, oven temperature was fixed at $70^{\circ} \mathrm{C}$ for one minute, then increasing up to $325^{\circ} \mathrm{C}$ by $10^{\circ} \mathrm{C} /$ min gradient and held for 10 minutes at $325{ }^{\circ} \mathrm{C}$. Total separation time was 37.5 minutes. The MS detector was performed at EI mode. Data acquisition was performed in full scan mode with mass range between 50-650 m/z.

\subsection{GC/MS data processing}

All raw data files obtained from GC/MS were analyzed in MS-DIAL metabolomics and lipidomics platform (http:// prime.psc.riken.jp/Metabolomics_Software/MS-DIAL/). In MS-DIAL settings, mass range was between 0-1000 Da. Minimum peak height for peak detection was 1000 amplitude. Retention time tolerance was $1 \mathrm{~min}$. Identification score cut off was $70 \%$. For metabolite identification we used GC/MS all public Kovats retention index data base (15302 records) (http:// prime.psc.riken.jp/Metabolomics_Software/MS-DIAL/) Peak areas of metabolites were calculated in MS-DIAL and normalized according to total ion chromatogram. In statistic analysis, we used excel macro (http:// prime.psc.riken.jp/Metabolomics_Software/StatisticalAnalysisOnMicrosoftExcel/index.html).

Principal component analysis (PCA) was carried out to understand the effect of ofloxacin on metabolome structure of E. coli. T-test was used to find differentially expressed metabolites under ofloxacin stress $(\mathrm{p}<0.05) \quad$ Differentially expressed metabolites were evaluated in pathway analysis (https://www.metaboanalyst.ca/faces/upload/PathUploadView.xhtml). Pathway analysis was carried out by using Metaboanalyst metabolomics platform (https://www.metaboanalyst.ca) and KEGG database system (https://www.genome.jp/kegg/ pathway.html).

Acknowledgements: There was no financial support fort his study.

Author contributions: Concept - E.K.; Design - E.K., C.O.; Supervision - E.K.; Materials - C.O.; Data Collection and/or Processing - E.K., C.O.; Analysis and/or Interpretation - E.K.; Literature Search - C.O.; Writing - E.K., C.O.; Critical Reviews - E.K., C.O.

Conflict of interest statement: The authors declared no conflict of interest.

\section{REFERENCES}

[1] Alos JI. Antibiotic resistance: A global crisis]. Enferm Infecc Microbiol Clin. 2015; 33(10): 692-699. [CrossRef].

[2] Ventola CL. The antibiotic resistance crisis: part 2: management strategies and new agents. P T.2015; 40(5): 344-352 
[3] Caudle WM, Bammler TK, Lin Y, Pan S, Zhang J. Using 'omics' to define pathogenesis and biomarkers of Parkinson's disease. Expert Rev Neurother. 2010; 10(6): 925-942. [CrossRef]

[4] Hasin Y, Seldin M, Lusis A. Multi-omics approaches to disease. Genome Biol. 2017; 18(1): 83-92. [CrossRef]

[5] Sacco F, Silvestri A, Posca D, Pirro S, Gherardini PF, Castagnoli L, Mann M, Cesareni G. Deep Proteomics of Breast Cancer Cells Reveals that Metformin Rewires Signaling Networks Away from a Pro-growth State. Cell Syst. 2016; 2(3): 159-171. [CrossRef]

[6] Li W, Zhang S, Wang X, Yu J, Li Z, Lin W, Lin X. Systematically integrated metabonomic-proteomic studies of Escherichia coli under ciprofloxacin stress. J Proteomics. 2018; 179(1): 61-70. [CrossRef]

[7] Patkari M, Kumbhar C, Nag A, Mehra S. Distinct transcriptomic response of S. coelicolor to ciprofloxacin in a nutrient-rich environment. Appl Microbiol Biotechnol. 2018; 102(24): 10623-10643. [CrossRef]

[8] Wright MS, Suzuki Y, Jones MB, Marshall SH, Rudin SD, van Duin D, Kaye K, Jacobs MR, Bonomo RA, Adams MD. Genomic and transcriptomic analyses of colistin-resistant clinical isolates of Klebsiella pneumoniae reveal multiple pathways of resistance. Antimicrob Agents Chemother. 2015; 59(1): 536-543. [CrossRef]

[9] Li H, Zhang DF, Lin XM, Peng XX. Outer membrane proteomics of kanamycin-resistant Escherichia coli identified MipA as a novel antibiotic resistance-related protein. FEMS Microbiol Lett. 2015; 362(11): 1-8. [CrossRef]

[10] Griffin JL, Atherton H, Shockcor J, Atzori L. Metabolomics as a tool for cardiac research. Nature Reviews Cardiology 2011; 8(11): 630-643. [CrossRef]

[11] Fukusaki E. Application of Metabolomics for High Resolution Phenotype Analysis. Mass Spectrom. 2014; 3(3): 1-8. [CrossRef]

[12] Vincent IM, Ehmann DE, Mills SD, Perros M, Barrett MP. Untargeted Metabolomics To Ascertain Antibiotic Modes of Action. Antimicrob Agents Chemother. 2016; 60(4): 2281-2291. [CrossRef]

[13] Zampieri M, Zimmermann M, Claassen M, Sauer U. Nontargeted Metabolomics Reveals the Multilevel Response to Antibiotic Perturbations. Cell Reports. 2017; 19(6): 1214-1228. [CrossRef]

[14] Xiong XY, Sheng XQ, Liu D, Zeng T, Peng Y, Wang YC. A GC/MS-based metabolomic approach for reliable diagnosis of phenylketonuria. Anal Bioanal Chem. 2015; 407(29): 8825-8833. [CrossRef]

[15] Blondeau JM. Fluoroquinolones: Mechanism of action, classification, and development of resistance. Surv Ophthalmol. 2004; 49(1): 73-78. [CrossRef]

[16] Hooper DC. Mechanisms of action of antimicrobials: Focus on fluoroquinolones. Clinical Infectious Diseases.2001; 32(1): 9-15. [CrossRef]

[17] CLSI. Methods for Dilution Antimicrobial Susceptibility Tests for Bacteria That Grow Aerobically; Approved Standard -Tenth Edition.CLSI document M07-A10. Wayne, PA: Clinical and Laboratory Standards Institute. 2005

[18] Nemutlu E, Zhang S, Xu YZ, Terzic A, Zhong L, Dzeja PD, Cha YM. Cardiac Resynchronization Therapy Induces Adaptive Metabolic Transitions in the Metabolomic Profile of Heart Failure. J Card Fail. 2015; 21(6): 460-469. [CrossRef]

[19] Rosato RR, Fernandez R, Paz LI, Singh CR, Rosato AE. TCA Cycle-Mediated Generation of ROS Is a Key Mediator for HeR-MRSA Survival under beta-Lactam Antibiotic Exposure. Plos One. 2014; 9(6): 1-10. [CrossRef]

[20] Lorenz MC, Fink GR. The glyoxylate cycle is required for fungal virulence. Nature. 2001; 412(6842): 83-86. [CrossRef]

[21] Jung J, Park W. Comparative genomic and transcriptomic analyses reveal habitat differentiation and different transcriptional responses during pectin metabolism in Alishewanella species. Appl Environ Microbiol. 2013; 79(20): 6351-6361. [CrossRef]

[22] Kohanski MA, Dwyer DJ, Hayete B, Lawrence CA, Collins JJ. A common mechanism of cellular death induced by bactericidal antibiotics. Cell. 2007; 130(5): 797-810. [CrossRef]

[23] Nandakumar M, Nathan C, Rhee KY. Isocitrate lyase mediates broad antibiotic tolerance in Mycobacterium tuberculosis. Nat Commun. 2014; 5(1): 4306-4316. [CrossRef]

[24] Ahn S, Jung J, Jang IA, Madsen EL, Park W. Role of Glyoxylate Shunt in Oxidative Stress Response. J Biol Chem. 2016; 291(22): 11928-11938. [CrossRef]

[25] Cameron JC, Pakrasi HB. Glutathione facilitates antibiotic resistance and photosystem I stability during exposure to gentamicin in cyanobacteria. Appl Environ Microbiol.2011; 77(10): 3547-3550. [CrossRef] 
[26] Cameron JC, Pakrasi HB Essential role of glutathione in acclimation to environmental and redox perturbations in the cyanobacterium Synechocystis. Plant Physiol. 2010; 154(4): 1672-1685. [CrossRef]

[27] Iyer R, Delcour AH. Complex inhibition of OmpF and OmpC bacterial porins by polyamines. J Biol Chem. 1997; 272(30): 18595-18601.

[28] Sarathy JP, Lee E, Dartois V. Polyamines inhibit porin-mediated fluoroquinolone uptake in mycobacteria. PLoS One 2013; 8 (6): 1-10. [CrossRef]

[29] Parsons JB, Rock CO Is bacterial fatty acid synthesis a valid target for antibacterial drug discovery? Curr Opin Microbiol. 2011; 14(5): 544-549. [CrossRef]

[30] Wright HT, Reynolds KA. Antibacterial targets in fatty acid biosynthesis. Curr Opin Microbiol. 2007; 10(5): 447-453. [CrossRef]

[31] Dunnick JK, O'Leary WM. Correlation of bacteria lipid composition with antibiotic resistance. J Bacteriol. 1970; 101(3): 892-900.

[32] Kuiukina MS, Ivshina IB, Rychkova MI, Chumakov OB. Effect of composition of cellular lipids of formation of nonspecific antibiotic resistance of alkanotrophic rhodocoocci. Mikrobiologiia. 2000; 69(1): 62-69.

This is an open access article which is publicly available on our journal's website under Institutional Repository at http://dspace.marmara.edu.tr. 